\title{
Filigrane
}

Écoutes psychothérapiques

\section{Une écluse entre cinéma et psychanalyse. Recension du livre Remake, de Jean Imbeault \\ Jean Imbeault, 2012, Remake, Paris, penser/rêver, Éd. de l'Olivier}

\section{André Jacques}

Volume 22, numéro 2, automne 2013

Psychanalyse et temporalités II

URI : https://id.erudit.org/iderudit/1022561ar

DOI : https://doi.org/10.7202/1022561ar

Aller au sommaire du numéro

Éditeur(s)

Revue Santé mentale au Québec

ISSN

1192-1412 (imprimé)

1911-4656 (numérique)

Découvrir la revue

Citer ce compte rendu

Jacques, A. (2013). Compte rendu de [Une écluse entre cinéma et psychanalyse. Recension du livre Remake, de Jean Imbeault / Jean Imbeault, 2012, Remake, Paris, penser/rêver, Éd. de l'Olivier]. Filigrane, 22(2), 115-117.

https://doi.org/10.7202/1022561ar 


\section{Une écluse entre cinéma et psychanalyse. Recension du livre Remake, de Jean Imbeault ${ }^{1}$}

\section{André Jacques}

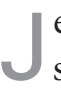

ean Imbeault amorce son livre Remake par une scène du film La neuvaine se déroulant sur le bord du fleuve Saint-Laurent. Il le termine sur une note mélancolique en relatant un souvenir personnel où il est lui-même sur la rive par une journée grise. "Arrivé à Baie-Comeau vers dix-sept heures. Marché jusqu'au quai. Attendu la tombée du jour. Assis face au fleuve...» Entre ce début et cette fin, l'eau coule, des films passent tels des paquebots ou des cargos dont il est à la fois le spectateur, le descripteur et le passager songeur et envoûté.

En cours de récit, Imbeault cite Roland Barthe (dans $S / Z$ ), pour qui un bon conteur trouve toujours le moyen de construire une «écluse qui ajuste les deux niveaux (le réel et l'irréel), que l'écluse rend communément "navigable” ». Et c'est justement cette sorte de construction que nous offre ce livre passionnant qui nous convie à une "navigation commune » entre cinéma et psychanalyse. Par un jeu subtil de portes et de vannes, d'associations et de rapprochements parfois surprenants, Imbeault fait se rejoindre ces deux surfaces liquides ayant en commun le même pouvoir immersif: on peut être « en cinéma » comme on est " en analyse », et circuler librement de l'un à l'autre, pour peu que l'on s'ouvre à leur commune fluidité.

Dans le vocabulaire usuel de tout cinéaste, le mot remake désigne un film reproduisant avec de nouveaux acteurs la première version d'un film à succès. Dans le monde cinématographique, remake évoque tantôt les pièges de la redondance, tantôt le pactole d'une relance opérée pour des motifs la plupart du temps bien loin de l'inspiration créatrice. Pour un psychanalyste, remake ouvre un vaste champ sémantique allant de l'après-coup au transfert, en passant par la répétition, l'identification et diverses formes de la projection. 
Les textes que chapeaute ce titre sont la reproduction du «journal» qu'Imbeault a tenu et dont il a publié de larges extraits dans la revue penser/rêver entre 2007 et 2011. Cette revue, pour ceux qui la connaîtraient peu ou pas du tout, a été fondée en 2002 par une équipe dont plusieurs membres avaient présidé à l'" inachèvement» de la Nouvelle Revue de psychanalyse (NRP) à l'automne de 1994. Dirigée par Michel Gribinski, penser/rêver constitue une sorte d'héritière de la NRP, elle-même fondée en 1970 par le regretté Jean-Bertrand Pontalis, ouverte à la littérature et aux arts autant qu'à divers mouvements psychanalytiques et dirigée par le co-auteur du célèbre Vocabulaire de la psychanalyse tout au long de ses cinquante numéros.

Le livre d'Imbeault comporte onze «entrées " portant chacune le titre d'un film: La neuvaine (de Bernard Émond); The Grifters (de Stephen Frears); Le fils (des frères Dardenne); Van Gogh (de Maurice Pialat); Gegen die Wand (en anglais: Head-on, de Fatih Akin); Monsieur Klein (de Joseph Losey), Paranoid Park (de Gus Van Sant); Two Lovers (de James Gray); Le Guépard (de Luchino Visconti); Far from Heaven (de Todd Haynes, remake de All That Heaven Allows, de Douglas Sirk); Le mépris (de Jean-Luc Godard).

Chacune des onze entrées relate les circonstances où l'auteur a vu ou revu le film et ses associations à l'égard du film lui-même et de ce qui a pour lui entouré son visionnement. Tôt ou tard dans chaque entrée se retrouve un synopsis souvent saisissant du film. Puis, en points et contre-points, Imbeault présente des liens audacieux et personnalisés avec sa pratique de la psychanalyse et le fondement de celle-ci : les textes de Sigmund Freud. «Ce journal, écrit Imbeault, est celui d'un analyste inquiet, mal assuré, jamais certain du terrain sur lequel il s'avance. Il y a toujours eu pour moi un lien, plus ou moins avouable, entre voir des films, faire de l'analyse, écrire dans le sillage de certains films que j'ai vus, et m'entêter à rendre compte, à ma manière, de ce que j'essaie de faire quand je fais de l'analyse» (92).

C'est dans ce sens qu'il rapproche le fait d' «être en analyse» de celui d' «être en cinéma» (comme on dit «être en voyage»). Ni en analyse, ni en cinéma n'est-il « uniquement spectateur, ni entraîné dans une cogitation, ni encadré dans une démonstration théorique». Ni l'un ni l'autre n'est «une contemplation, un ravissement, une extase, un transport hors de soi ou du monde. [...] Être en cinéma, autant qu'être en analyse, écrit-il, est un état prosaïque. C'est une relation - singulière, il est vrai - à la réalité. Une relation à la réalité qui ne peut s'établir et se rétablir qu'à même la reprise perpétuelle d'une première expérience » (127-128). Cette première expérience, sans doute à jamais perdue, le spectateur autant que l'analysant en porte en lui la trace 
vers laquelle il revient sans cesse et qui lui sert de balise autant que d'horizon. "Le cinéma, c'est ce qui nous revient après être passé par l'oubli», cite Imbeault d'un auteur dont il a oublié le nom. Or, poursuit-il, «ce qui nous revient», ce n'est pas le passé, mais le «droit chemin » de notre présent.

Mais la psychanalyse ne peut nous aider à retrouver ce droit chemin du présent, si on s'en est égaré, que si on en a absolument besoin et qu'on ne voit que la psychanalyse pour nous ramener à la réalité. Cette exigence est celle-là même du «vrai » cinéaste, le distinguant d'un «non-cinéaste ». Comme l'écrit Martin Scorsese, qu'Imbeault cite à partir de Voyage à travers le cinéma américain, «le cinéaste est celui qui a besoin du cinéma, qui en a un besoin absolu, pour percevoir, pour saisir, pour voir les choses telles qu'elles sont, pour revenir au réel. Il est donc toujours en lutte. En lutte contre ceux qui tentent de lui faire dire ce qu'il n'a pas voulu dire. En lutte contre les forces qui l'enjoignent - du dedans comme du dehors - de montrer le contraire de ce qu'il voit» (183).

L'analysant ne se sert-il pas de l'analyse dans le même souci de vérité, sur fond de la même nécessité intérieure? Imbeault n’a de cesse de rapprocher l'éthique cinématographique de celle de la psychanalyse. Et de poser le cinéma à titre de "succédané privilégié » du monde onirique. Or, écrit-il, «pas d'appartenance possible au monde humain sans la perfusion insensible, dans ma conscience, de ces succédanés du rêve que sont identification, idéalisation, suggestion, hypnose, sentiment social, passion amoureuse, névrose » (146), dont le cinéma présente un remarquable condensé.

Le jeu d'écluse de Remake entre le cinéma et la psychanalyse profite également à l'un et à l'autre. Dans une langue claire, la «langue commune » chère à Pontalis, Jean Imbeault présente une vision de la psychanalyse et du cinéma qui tout à la fois les rapproche, les spécifie et montre avec une sobre élégance comment et combien l'un et l'autre sont issus d'une même source.

André Jacques

jacques.andre@uqam.ca

\section{Note}

Jean Imbeault, 2012, Remake, Paris, penser/rêver, Éd. de l'Olivier. 